International Journal of Pure and Applied Mathematics

Volume 98 No. 3 2015, 351-353

ISSN: 1311-8080 (printed version); ISSN: 1314-3395 (on-line version)

url: http://www.ijpam.eu

doi: http://dx.doi.org/10.12732/ijpam.v98i3.7

ijpam.eu

\title{
ZERO-DIMENSIONAL SCHEMES CONTAINED BETWEEN TWO CONSECUTIVE MULTIPLE POINTS
}

\author{
E. Ballico \\ Department of Mathematics \\ University of Trento \\ 38123 Povo (Trento) - Via Sommarive, 14, ITALY
}

\begin{abstract}
Fix an integral projective variety, $P \in X_{\text {reg }}, m>0, L \in \operatorname{Pic}(X)$. Let $W \subseteq H^{0}(X, L)$ be a linear subspace. Set $b:=\operatorname{dim}(W(-m P))$ and $a:=$ $\operatorname{dim}(W(-(m+1) P))$. Fix an integer $z$ such that $0 \leq z \leq b-a$. We prove the existence of a zero-dimensional scheme $Z \subset X$ such that $m P \subseteq Z \subseteq(m+1) P$, $\operatorname{deg}(Z)=\operatorname{deg}(m P)+z$ and $\operatorname{dim}(W(-Z))=b-z$.
\end{abstract}

AMS Subject Classification: 14N05

Key Words: fat point, zero-dimensional scheme, multiple point

Let $X$ be an integral projective variety with $\operatorname{dim}(X)>0$ over an algebraically closed base field $K$. Fix $P \in X$. For each integers $m>0$ let $m P$ be the closed subscheme of $X$ with $\left(\mathcal{I}_{X}\right)^{m}$ as its ideal sheaf. For each line bundle $R$ on $X$, any linear subspace $W \subseteq H^{0}(X, L)$ and any zero-dimensional scheme $Z \subset X$ set $W(-Z):=W \cap H^{0}\left(X, \mathcal{I}_{Z} \otimes L\right)$. In this note we prove the following result.

Received: September 19, 2014

(c) 2015 Academic Publications, Ltd. url: www.acadpubl.eu 
Theorem 1. Fix an integral projective variety, $P \in X_{r e g}, m>0, L \in$ $\operatorname{Pic}(X)$. Let $W \subseteq H^{0}(X, L)$ be a linear subspace. Set $b:=\operatorname{dim}(W(-m P))$ and $a:=\operatorname{dim}(W(-(m+1) P))$. Fix an integer $z$ such that $0 \leq z \leq b-a$. Then there is a zero-dimensional scheme $Z \subset X$ such that $m P \subseteq Z \subseteq(m+1) P$, $\operatorname{deg}(Z)=\operatorname{deg}(m P)+z$ and $\operatorname{dim}(W(-Z))=b-z$.

Theorem 1 cannot be extended to scheme contained between $m P$ and $(m+$ 2) $P$, even if $X$ is a smooth curve, $m=1$ and $W=H^{0}(X, L)$, because we fixed the point $P$ and hence it may be an ordinary flex of the linear system $|L|$. In the case $m=1$ when we take general $P$, see [1], [2] and [3].

Proof of Theorem 1. We may assume $n:=\operatorname{dim}(X)>0$ and $b-a>0$. Fix a regular sequence $x_{1}, \ldots, x_{n}$ of the local $\operatorname{ring} \mathcal{O}_{X, P}$ and call $\mathbf{m}$ the maximal ideal of $\mathcal{O}_{X, P}$. Let $\mathcal{J}$ be the ideal sheaf of $m P$ in $(m+1) P$. We have $\mathcal{O}_{(m+1) P} \cong$ $K\left[x_{1}, \ldots, x_{n}\right] /\left(x_{1}, \ldots, x_{n}\right)^{m+1}$ and $\mathcal{O}_{m P} \cong K\left[x_{1}, \ldots, x_{n}\right] /\left(x_{1}, \ldots, x_{n}\right)^{m}$. Therefore $\mathcal{J}$ is a $K$-vector space with as a basis the set $\mathcal{B}$ of all monomials of degree $m+1$ in the variables $x_{1}, \ldots, x_{n}$ (a $K$-vector space of dimension $\left(\begin{array}{c}n+m \\ n-1\end{array}\right)$ ). For any $E \subseteq \mathcal{B}$ let $\langle E\rangle$ be the $K$-linear subspace of $\mathcal{J}$ generated by $E$. Since $\mathcal{J}^{2}=0$ each $E \subseteq \mathcal{B}$ define a zero-dimensional scheme $Z_{E} \subseteq(m+1) P \subset X$ such that $Z_{E} \supseteq m P$ and $\operatorname{deg}\left(Z_{E}\right)=\operatorname{deg}(m P)+\sharp(E)$. Note that $b-a \leq\left(\begin{array}{c}n+m \\ n-1\end{array}\right)$ and hence $z \leq\left(\begin{array}{c}n+m \\ n-1\end{array}\right)$. We will find $Z=Z_{E}$ for some $E$. If $z=0$, then take $Z=m P$, i.e. $E=\emptyset$. Hence we may assume $z>0$ and use induction on $z$ for the existence of $Z$ of the form $Z_{E}$. Take $F \subset \mathcal{B}$ such that $\sharp(F)=z-1$ and $\operatorname{dim}\left(W\left(-Z_{F}\right)\right)=a-z+1$. Since $a-z+1>b$ and $\mathcal{B}$ is a basis of the $K$-vector space $\mathcal{B}$ there is $m \in \mathcal{B} \backslash F$ and $\left.f \in W\left(-Z_{F}\right)\right)$ such that $f \mid m \neq 0$. Take $E:=F \cup\{m\}$.

Corollary 1. Fix an integral projective variety of dimension $n>0, L \in$ $\operatorname{Pic}(X)$ and a linear subspace $W \subseteq H^{0}(X, L)$ be a linear subspace. Fix integers $s>0$ and $m_{i}>0,1 \leq i \leq s$. Suppose that for general $P_{1}, \ldots, P_{s} \in X_{\text {reg }}$ we have $\operatorname{dim}\left(W\left(-\cup_{i} m_{i} P_{i}\right)\right)=\operatorname{dim}(W)-\sum_{i}\left(\begin{array}{c}m_{i}+n-1 \\ n\end{array}\right)$ and $W\left(-\cup_{i}\left(m_{i}+1\right) P_{i}\right)=0$. Then for general $O_{1}, \ldots, O_{s} \in X_{\text {reg }}$ there are schemes $Z_{i}, 1 \leq i \leq s$, such that $m_{i} P_{i} \subseteq Z_{i} \subseteq\left(m_{i}+1\right) P_{i}$ for all $i, \sum_{i} \operatorname{deg}\left(Z_{i}\right)=\operatorname{dim}(W)$ and $W\left(-\cup_{i} Z_{i}\right)=0$.

Now we drop the assumption that $K$ is algebraically closed. Easy examples show that there is no hope of a straightforward extension if $P \notin X(K)$, i.e. if the natural map $K \rightarrow \mathcal{O}_{X, P} / \mathbf{m}$ is not an isomorphism. If we may find a regular sequence defined $x_{1}, \ldots, x_{n}$ defined over $K$, then the proof just given works verbatim. It exists, by Nakayama's lemma, if we assume that $P$ is a smooth point of $X$. The definition of the schemes $Z_{E}, E \subseteq \mathcal{B}$ depends from the choice of the regular sequence. If we fix we get the following corollary of the proof of 
Theorem 1

Corollary 2. Fix an integral projective variety, $P \in X_{\text {reg }}, m>0, L \in$ $\operatorname{Pic}(X)$. Let $W \subseteq H^{0}(X, L)$ be a linear subspace. Fix a regular system of parameters $x_{1}, \ldots, x_{n}$ and use it to define $\mathcal{B}$ and all $Z_{E}, E \subseteq \mathcal{B}$. Fix $E \subseteq F \subseteq \mathcal{B}$ and set $a:=\operatorname{dim}\left(W\left(-Z_{F}\right)\right)$ and $b:=\operatorname{dim}\left(W\left(-Z_{E}\right)\right)$. Fix an integer $z$ such that $0 \leq z \leq b-a$. Then there is $G$ such that $E \subseteq G \subseteq F, \sharp(G)=\sharp(E)+z$ and $\operatorname{dim}\left(W\left(-Z_{G}\right)\right)=b-z$.

If we have a way to fix a regular system of parameters for all points in a open subset $U$ of $X$ (e.g. if $U$ is an open subset of $\mathbb{A}^{n}$ with coordinates $z_{1}, \ldots, z_{n}$ and we take $\left(z_{1}-a_{1}, \ldots, z_{n}-a_{n}\right)$ at $\left.P=\left(a_{1}, \ldots, a_{n}\right)\right)$, then we are in the set-up of $[2]$.

Remark 1. Take $P \in \operatorname{Sing}(X)$ and call $\mathbf{m}$ the maximal ideal of $\mathcal{O}_{X, P}$. Take $m>0$ such that for $t=m, m+1$ the natural map $S^{t}\left(\mathbf{m} / \mathbf{m}^{2}\right) \rightarrow \mathbf{m}^{t} / \mathbf{m}^{t+1}$ is an isomorphism (this condition is always satisfied if $m=1$ ). Then Theorem 1 is true for $P$.

\section{Acknowledgements}

The author was partially supported by MIUR and GNSAGA of INdAM (Italy).

\section{References}

[1] M.C. Brambilla, G. Ottaviani, On partial polynomial interpolation, Linear Algebra Appl. 435, No. 6 (2011), 1415-1445.

[2] E. Ballico, A partial multivariate polynomial interpolation problem, Int. Math. Forum, 2, No. 61-64 (2007), 3089-3094.

[3] The defectivity of zero-dimensional subschemes contained in double points, Int. J. Contemp. Math. Sci., 2, No. 29-32 (2007), 1545-1548. 
